\title{
The chronic challenge-new vistas on long-term multisite contacts to the central nervous system
}

\author{
Ulrich G. Hofmann ${ }^{1,2 *}$ and Jürgen Krüger ${ }^{3}$ \\ ${ }^{1}$ Section for Neuroelectronic Systems, Clinic for Neurosurgery, Albert-Ludwigs-University Freiburg, Freiburg, Germany, \\ ${ }^{2}$ Cluster of Excellence "BrainLinks-BrainTools" EXC 1086, Freiburg, Germany, ${ }^{3}$ A G Hirnforschung, Universität Freiburg, \\ Freiburg, Germany
}

Keywords: multisite neuronal recording, gliosis, indwelling implants, compliance match hypothesis, flexible microprobes, hydrogel coating, polymeric microelectrodes

This Special Research Topic at hand is a collection of contributions from eminent research groups shedding light on several aspects of the still unresolved problem of a truly chronic cortical interface to enable long term brain-machine interfacing to human patients.

The hypothesis article of Fernandez et al. (2014) adds to the three generally agreed-on features for biocompatibility (bio-safety, bio-stability, and bio-functionality) with a fourth one that mirrors the demand for "bio-tolerability." Sommakia et al. (2014a) study aims to reduce the almostimmediate adsorption of non-cellular tissue components upon insertion by dip-coating polyethylene glycol (PEG) as a "stealth" cover. It points toward a beneficial alteration of adsorption on the probe, but cautions PEG's immediate use for long term implants in the brain. In fact, in a second contribution based on a mixed-brain culture (Sommakia et al., 2014b), they show evidence for a complex response of glia cells on micro-wires dip-coated with PEG and/or lipopolysaccharides (LPS), but not of neurons, which is somewhat contradictory to pure in vivo findings.

In the same context of passive probe coatings, De Faveri et al. (2014) moved toward a more "natural" method by coating glass-insulated micro-wires with fibrin hydrogel, as a biological cushion between brain and probe. Using immunofluorescence techniques, they were able to demonstrate a beneficial effect on longer term astrocytic responses and successful encapsulation of brain cells in fibrin as in Richter (2012).

Beyond the modulatory effects of passive surface coatings, two articles review organic coatings

Edited and reviewed by: Laura Ballerini,

University of Trieste, Italy

*Correspondence:

Ulrich G. Hofmann, ulrich.hofmann@coregen. uni-freiburg.de

Received: 18 February 2015 Accepted: 27 February 2015 Published: 18 March 2015

Citation: Hofmann UG and Krüger J (2015) The chronic challenge-new vistas on long-term multisite contacts to the central nervous system.

Front. Neuroeng. 8:3. doi: 10.3389/fneng.2015.00003 for micro-contacts in the nervous system. The contribution by Asplund et al. (2014) concisely reviews electrodes based on conductive polymers, not only for improving site-tissue coupling, but also for electrically eluting anti-inflammatory drugs using various stimulation patterns. They go to great depth on how to apply this elution process to a living being, since the active elution technique inherently requires compliance with demanding bio-compatibility issues.

The review of Aregueta-Robles et al. (2014) addresses the topic of organic and nanoscopic coatings with a wider perspective, and thus provides an excellent overview regarding a huge variety of the various reported approaches including the "living electrode" of Ochiai et al. (1980) and their adaptation by Richter et al. (2010, 2011).

Extending the time frame of all above mentioned studies, Prasad et al. (2014) investigate whether the brain's foreign body response is the sole cause for poor electrode yield using $\mathrm{Pt} / \mathrm{Ir}$ micro wire arrays. They state that leading aspects include the suboptimal construction of the micro wires, as well as the severing of the blood brain barrier upon insertion. In order to achieve a deeper insight into the suboptimal micro array construction, another study of the same group (Sankar et al., 2014) analyses long-term impedance spectra using FEM simulation, and concludes that the initial increase in electrode-tissue impedance in vivo can be attributed 
to cell attachment and gliosis on the micro wires. Furthermore they show that the long-term decrease is probably caused by de-lamination and cracks in the wire's insulation layer.

Even though the contribution of Castagnola et al. (2014) discusses the use of nano materials for improving the signal quality from brain micro-recordings as well, its main theme is the interpretation of the so called "compliance match hypothesis" (Stieglitz and Meyer, 1999) by making cortical interfaces softer reducing the permanent mismatch between rigid micro probes and the brain's softness. Obviously, comparing elastic moduli of brain and probe materials reveals a discrepancy of several orders of magnitude, and so the positive evidence of De Faveri et al. (2014) may be based on a compliance adaptation between both due to the fibrin layer. However, Castagnola et al. (2014) as well as Richter et al. (2013), Sohal et al. (2014) and Xie et al. (2014) all use substrate materials for their multisite arrays, which are still far away from brain's bulk modulus. More importantly, they are flexible ("soft" when compared to brain tissue) due to their geometrical setup. We hypothesize that this flexibility might be the reason for Krüger's 7 year recording record in the non-human primate using ultra-thin metal wires (Krüger et al., 2010). Castagnola et al. (2014) approaches a similar goal by electro-depositing microscopic spheres at the working end of very thin wires-intended for subarachnoidal recordings. Sohal et al. (2014) in contrast, intracortically implants a geometrically "wavy" structure and shows evidence for good recordings from a limited number of animals for up to 2 years. The histological findings from their and Richter's thin multisite implants depict a reduced gliosis, and thus corroborates the need for a matching

\section{References}

Aregueta-Robles, U. A., Woolley, A. J., Poole-Warren, L. A., Lovell, N. H., and Green, R. A. (2014). Organic electrode coatings for nextgeneration neural interfaces. Front. Neuroeng. 7:15. doi: 10.3389/fneng.2014. 00015

Asplund, M., Boehler, C., and Stieglitz, T. (2014). Anti-inflammatory polymer electrodes for glial scar treatment: bringing the conceptual idea to future results. Front. Neuroeng. 7:9. doi: 10.3389/fneng.2014.00009

Castagnola, E., Ansaldo, A., Maggiolini, E., Ius, T., Skrap, M., Ricci, D., et al. (2014). Smaller, softer, lower-impedance electrodes for human neuroprosthesis: a pragmatic approach. Front. Neuroeng. 7:8. doi: 10.3389/fneng.2014.00008

De Faveri, S., Maggiolini, E., Miele, E., De Angelis, F., Cesca, F., Benfenati, F., et al. (2014). Bio-inspired hybrid microelectrodes: a hybrid solution to improve longterm performance of chronic intracortical implants. Front. Neuroeng. 7:7. doi: 10.3389/fneng.2014.00007

Eleryan, A., Vaidya, M., Southerland, J., Badreldin, I. S., Balasubramanian, K., Fagg, A. H., et al. (2014). Tracking single units in chronic, large scale, neural recordings for brain machine interface applications. Front. Neuroeng. 7:23. doi: 10.3389/fneng.2014.00023

Fernandez, E., Greger, B., House, P. A., Aranda, I., Botella, C., Albisua, J., et al. (2014). Acute human brain responses to intracortical microelectrode arrays: challenges and future prospects. Front. Neuroeng. 7:24. doi: 10.3389/fneng.2014.00024

Krüger, J., Caruana, F., Dalla Volta, R., and Rizzolatti, G. (2010). Seven years of recording from monkey cortex with a chronically implanted multiple microelectrode. Front. Neuroeng. 3:6. doi: 10.3389/fneng.2010.00006

Ochiai, H., Shibata, H., Sawa, Y., and Katoh, T. (1980). "Living electrode" as a longlived photoconverter for biophotolysis of water. Proc. Natl. Acad. Sci. U.S.A. 77, 2442-2444. compliance and intracortical softness. Richter's probes are made from polyimide (Rubehn et al., 2010), and they explain in detail how to implant them with help of a simple removable support.

This removable support in turn provides additional value, as Xie et al. (2014) state. Their use of label-free, endoscopic, in vivo Optical Coherence Tomography by the very same fiber supporting implantation demonstrates the first optical online monitoring of deep indwelling processes. This method may provide new vistas on the traditional problem of foreign body response, and may therefore shorten the time we have to spend in the Trough of Disillusionment.

Whatever effort is required, and whichever ideas will finally prove optimal for reaching the Plateau of Productivity for brainmachine interfaces, we are thankful that the need for biotolerability and reliability of brain implants over a substantial period of time, has already sunk into the community. Eleryan et al.'s (2014) single neuronal signal tracking method demonstrates this spirit of pro-active optimism by providing a highly valuable algorithm for following the progress of an individual neuron's participation in information processing.

This Special Research Topic's collection won't be the final word with respect to chronic implants, but for now it sets a roadsign toward truly stable neuro-cortical interfaces that lie on the horizon.

\section{Acknowledgments}

We are indebted to Dr. Richard C. Pinnell for making our manuscript comprehensible.

Prasad, A., Xue, Q.-S., Dieme, R., Sankar, V., Mayrand, R. C., Nishida, T., et al. (2014). Abiotic-biotic characterization of $\mathrm{Pt} / \mathrm{Ir}$ microelectrode arrays in chronic implants. Front. Neuroeng. 7:2. doi: 10.3389/fneng. 2014.0000

Richter, A. (2012). Biologisierung Tiefenhirn-Stimulierender Implantate Durch Besiedelung Mit Adulten Glandulären Stammzellen. Ph.D. thesis, University of Lübeck.

Richter, A., Danner, S., Kruse, C., and Hofmann, U. G. (2010). Applying controlled shear stress on cell covered microprobes. Biomed. Tech. 55(Supp. 1), 4. doi: 10.1515/BMT.2010.678

Richter, A., Kruse, C., Moser, A., Hofmann, U. G., and Danner, S. (2011). Cellular modulation of polymeric device surfaces: promise of adult stem cells for neuro-prosthetics. Front. Neurosci. 5:114. doi: 10.3389/fnins.2011.0011

Richter, A., Xie, Y., Schumacher, A., Löffler, S., Kirch, R. D., Al-Hasani, J., et al. (2013). A simple implantation method for flexible, multisite microelectrodes into rat brains. Front. Neuroeng. 6:6. doi: 10.3389/fneng.2013.00006

Rubehn, B., Lewis, C., Fries, P., and Stieglitz, T. A. (2010). "Flexible shaft electrodes for transdural implantation and chronic recording," in Proceedings of the 15th Annual Conference of the IFESS, (Vienna: IFMBE), 4.

Sankar, V., Patrick, E., Dieme, R., Sanchez, J. C., Prasad, A., and Nishida, T. (2014). Electrode impedance analysis of chronic tungsten microwire neural implants: understanding abiotic vs. biotic contributions. Front. Neuroeng. 7:13. doi: 10.3389/fneng.2014.00013

Sohal, H. S., Jackson, A., Jackson, R., Clowry, G. J., Vassilevski, K., O’Neill, A., et al. (2014). The sinusoidal probe: a new approach to improve electrode longevity. Front. Neuroeng. 7:10. doi: 10.3389/fneng.2014.00010

Sommakia, S., Gaire, J., Rickus, J. L., and Otto, K. J. (2014a). Resistive and reactive changes to the impedance of intracortical microelectrodes can be mitigated with polyethylene glycol under acute in vitro and in vivo settings. Front. Neuroeng. 7:33. doi: 10.3389/fneng.2014.00033 
Sommakia, S., Rickus, J. L., and Otto, K. J. (2014b). Glial cells, but not neurons, exhibit a controllable response to a localized inflammatory microenvironment in vitro. Front. Neuroeng. 7:41. doi: 10.3389/fneng.2014.00041

Stieglitz, T., and Meyer, J. U. (1999). Implantable microsystems. Polyimidebased neuroprostheses for interfacing nerves. Med. Device Technol. 10, 28-30.

Xie, Y., Martini, N., Hassler, C., Kirch, R. D., Stieglitz, T., Seifert, A., et al. (2014). In vivo monitoring of glial scar proliferation on chronically implanted neural electrodes by fiber optical coherence tomography. Front. Neuroeng. 7:34. doi: 10.3389/fneng.2014.00034
Conflict of Interest Statement: The authors declare that the research was conducted in the absence of any commercial or financial relationships that could be construed as a potential conflict of interest.

Copyright (c) 2015 Hofmann and Krüger. This is an open-access article distributed under the terms of the Creative Commons Attribution License (CC BY). The use, distribution or reproduction in other forums is permitted, provided the original author(s) or licensor are credited and that the original publication in this journal is cited, in accordance with accepted academic practice. No use, distribution or reproduction is permitted which does not comply with these terms. 